\section{Krankenhausaufenthalt führt zu keinem finanziellen Vorteil}

Sie kommt zu dem Ergebnis, dass eine Verlegung einer Bewohnerin oder eines Bewohners bei einem akuten Ereignis weder für die Klinik noch für die Alteneinrichtung finanziell reizvoll ist. Beide Einrichtungen zahlen drauf. Als Forscherinnen sind wir überzeugt, dass die Versorgung akut erkrankter Bewohnerinnen und Bewohner im Altenheim von den Einrichtungen selbst in vielerlei Weise gefördert werden kann. Hierzu wurden spezifische Modelle entwickelt, die Einweisungen in ein Krankenhaus reduzieren können und den betroffenen Personen positiv entgegenkommen.

\section{Interventionsmodell "Aktuelle Ereignisse im Blick" hilft}

Das Projektteam entwickelte nach der Auswertung der Ergebnisse einen Leitfaden für Altenpflegeeinrichtungen, das Interventionsmodell „Aktuelle Ereignisse im Blick“. Dieses Arbeitsbuch geht auf die speziellen Bedürfnisse der Einrichtungen ein und gibt praktische Anleitungen zur Vermeidung von Krankenhausaufenthalten.

Bei der Erstellung des Arbeitsbuchs arbeiteten wir eng mit Pflegefachpersonen, Heimleitern, Fachärzten und Angehörigen zusammen. So ist es gelungen, besonders praxisbezogene Hilfestellungen zu geben. Es ist zu hoffen, dass möglichst viele Altenheime das Interventionsmodell nutzen, um ihren Bewohnerinnen und Bewohnern mehr Lebensqualität $\mathrm{zu}$ bieten sowie gleichzeitig Kosten zu sparen. In Kürze wird das Arbeitsbuch über die Homepage der Universität Witten/Herdecke erhältlich sein.

Das Projekt „Innovative Versorgung von akut erkrankten Bewohnern und Bewohnerinnen im Altenheim" wurde im Rahmen des vom Ministerium für Gesundheit, Emanzipation, Pflege und Alter des Landes Nordrhein-Westfalen (MGEPA) innerhalb des Europäischen Fonds für regionale Entwicklung NRW Ziel 2 Programms 2007 - 2013 (EFRE) gefördert.

Prof. Christel Bienstein

Präsidentin des DBfK

\title{
Gesetz zur Hospiz- und Palliativversorgung: Qualifizierung in „Palliative care“ nötig
}

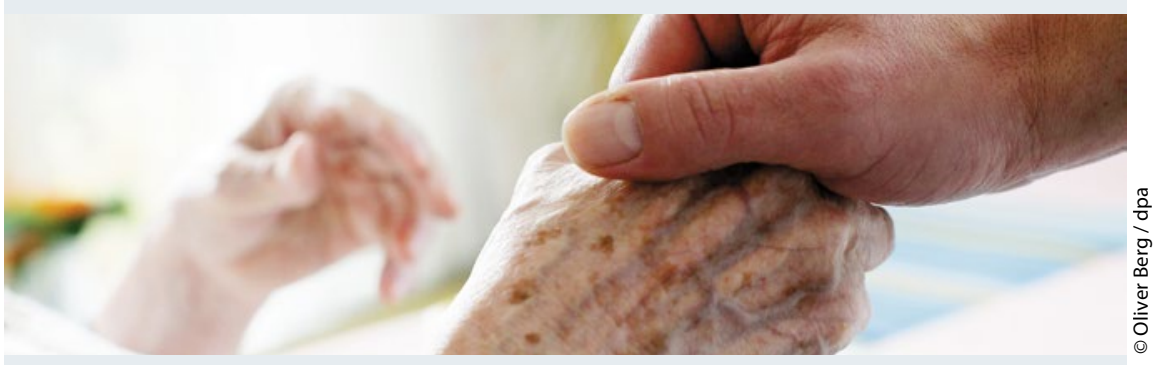

Das jetzt im Deutschen Bundestag verabschiedete Gesetz zur Verbesserung der Hospiz- und Palliativversorgung in Deutschland wurde vorab im Rahmen der Anhörung in Berlin von Experten gewürdigt. Die geplanten Verbesserungen wurden besonders in struktureller Hinsicht als gut und sinnvoll erachtet. Jedoch besteht einhellig die Meinung, dass das Gesetz den Bereich der allgemeinen Palliativversorgung im Krankenhaus sowie Regelungen für den erhöhten palliativen Pflegeaufwand in stationären Pflegeeinrichtungen nicht berücksichtigt. Nicht alle Menschen können, wollen und sollen in speziellen Einrichtungen sterben. Es reicht nicht aus, wie im Gesetz gefordert, dass jeder Menschen ein Anrecht auf Beratung hat, wie er in seiner letzten Lebensphase begleitet werden will, wenn keine Ressourcen vorhanden sind, die Wünsche entsprechend umzusetzen.

Es besteht somit dringender Handlungsbedarf, wenn man dem Leitsatz der Charta zur Versorgung schwerstkranker und sterbender Menschen folgt. Dieser lautet: „Jeder Mensch hat ein Recht auf ein Sterben unter würdigen Bedingungen". Hieraus sollte sich eine Verpflichtung zur Palliativversorgung sowohl in Krankenhäusern als auch in Pflegeheimen ergeben, der nur nachgekommen werden kann, wenn ausreichend Zeit und Personal, das heißt letztlich ausreichende finanzielle Ressourcen zur Verfügung gestellt werden.

Es ist wichtig, dass die Einrichtungen eine tragfähige Hospiz- und Palliativkultur entwickeln und leben, in die die Mitarbeiter einbezogen werden. Außerdem ist es zielführend, klare Qualitätsmerkmale aufzustellen und die Fragen der Organisation und Arbeitsabläufe im
Bedarfsfall zu klären. Die Zusammenarbeit und Vernetzung inner- und außerhalb des Krankenhauses /des Pflegeheimes müssen bekannt sein.

Zur Erreichung einer guten palliativen Versorgung und Betreuung auch außerhalb von Spezialeinrichtungen müssen jeweils mehrere Pflegefachkräfte in den Einrichtungen in "Palliative care" qualifiziert sein. Letztlich muss innen aber ausreichend Zeit in der täglichen Arbeit zur Begleitung von Menschen an ihrem Lebensende eingeräumt werden. Ein solches Vorgehen wäre nicht nur für Menschen in ihrer letzten Lebensphase von großem Vorteil, sondern würde auch zur beruflichen Zufriedenheit von Pflegekräften beitragen.

Wir wissen und sind fachlich in der Lage, Menschen in ihrer letzten Lebensphase würdig zu begleiten. $\mathrm{Ob}$ jedoch die palliative Versorgung im Krankenhaus als auch in Pflegeheimen über das Hospiz- und Palliativgesetz direkt geregelt werden kann, ist fraglich. Im Sinne aller Beteiligten muss jedoch um Finanzierungsmöglichkeiten gerungen werden. Hier steht der Vorschlag im Raum, einen OPS (Operationen- und Prozedurenschlüssel) zur Palliativbetreuung auf Allgemein- und Intensivstationen zu entwickeln. Für den Bereich der Pflegeheime schlägt der VKAD (Verband Katholische Altenhilfe in Deutschland e.V.) vor, zusätzlich zwei Vollstellen für Pflegefachkräfte je 100 Bewohner zu besetzen. Dies könnte dann unter Vorlage entsprechender Konzepte bei Pflegesatzverhandlungen geltend gemacht werden.

\section{Irmgard Menger}

Oberin i.R.,

Mitglied im Deutschen Pflegerat 